\title{
Differential impact of inflammasome-induced extracellular vesicles on immune cells: key control by the functional state of the recipient cell
}

\author{
Claudia Verderio $^{1}$, Marta Lombardi ${ }^{1}$, Francesco di Virgilio ${ }^{2}$ \\ ${ }^{1}$ CNR Institute of Neuroscience, U28 Building Via Follereau 3, Vedano al Lambro (MB), Italy; ${ }^{2}$ Department of Medical Sciences, University of \\ Ferrara, Italy \\ Correspondence to: Claudia Verderio. CNR instituite of Neuroscience, Università Milano-Bicocca, U28 Building Via Follereau 3, 20854 Vedano al \\ Lambro (MB), Italy. Email: c.verderio@in.cnr.it. \\ Comment on: Budden CF, Gearing LJ, Kaiser R, et al. Inflammasome-induced extracellular vesicles harbour distinct RNA signatures and alter \\ bystander macrophage responses. J Extracell Vesicles 2021;10:e12127.
}

Received: 05 October 2021; Accepted: 22 October 2021; Published: 30 November 2021.

doi: 10.21037/exrna-21-17

View this article at: https://dx.doi.org/10.21037/exrna-21-17

Inflammasomes are cytoplasmic multiprotein complexes that survey the cytosol of immune cells for pathogen contamination or cellular perturbation by sensing stimuli that activate an inflammatory response and integrating their downstream signaling (1). They act as innate immune sensors and, in response to pathogens/cell damage, trigger pyroptosis, a lytic form of programmed cell death.

The term "inflammasome" has been originally coined by Martinon et al. in 2002 who reported the assembly of cytosolic complexes which lead to proteolytic activation of caspases and inflammation (2).

Inflammasomes consist of a sensor protein (NLRC4, NLRP or AIM2 protein), an inflammatory caspase, and in some cases an adaptor protein, such as ASC (the adaptor molecule apoptosis-associated speck-like protein containing a CARD). Inflammasomes are activated by a multiplicity of pathogen-derived or endogenous factors, often in an inflammasome-specific fashion (e.g., NLRP1 or NLRC4 activation by anthrax lethal toxin or bacterial flagellin, respectively). The inflammasome subtype most frequently involved in inflammation is NLRP3, typically activated by agents that form plasma membrane pores (e.g., nigericin or extracellular ATP) and thus cause a large intracellular $\mathrm{K}^{+}$efflux. Once assembled and activated, the NLRP3 inflammasome triggers pro-caspase- 1 cleavage, which generates the enzymatically active caspase- 1 that in turn drives the enzymatic activation of the pro-inflammatory cytokines IL-1 $\beta$ and IL-18, as well as of gasdermins, a family of pore-forming effector proteins (Figure 1). Gasdermins have a homologous N-terminal gasdermin domain, which upon proteolytic removal of the inhibitory C-terminal domain forms a pore in the plasma membrane that causes membrane permeabilization, cell swelling and pyroptosis (3).

Appropriate inflammasome activation is vital for the immune cells to cope with pathogens or tissue damage, but aberrant inflammasome activation is associated to uncontrolled tissue responses and various inflammatory diseases. Some inflammasome sensors, such as NLRP3, have been identified as drivers of chronic inflammatory pathologies. In particular, gain-of-function mutations in NLRP3 inflammasomes cause cryopyrin-associated periodic syndromes (CAPS) that are characterized by chronic systemic inflammation.

Extracellular NLRP3 inflammasome oligomeric particles have been previously identified in the supernatant of activated macrophages and in the blood of CAPS patients (4).

It has long been known that one of the functional consequence of inflammasome activation is the release of extracellular vesicles $(\mathrm{EVs})$ from immune cells $(5-8)$. EVs are heterogeneous membrane structures released by all cell types, which contain and transfer cellular components from a donor to a recipient cell and act as potent mediators of cell-to-cell communication. Immune cells-derived EVs, i.e., released by monocytes, microglia, dendritic cells and macrophages, were originally reported to serve as an 


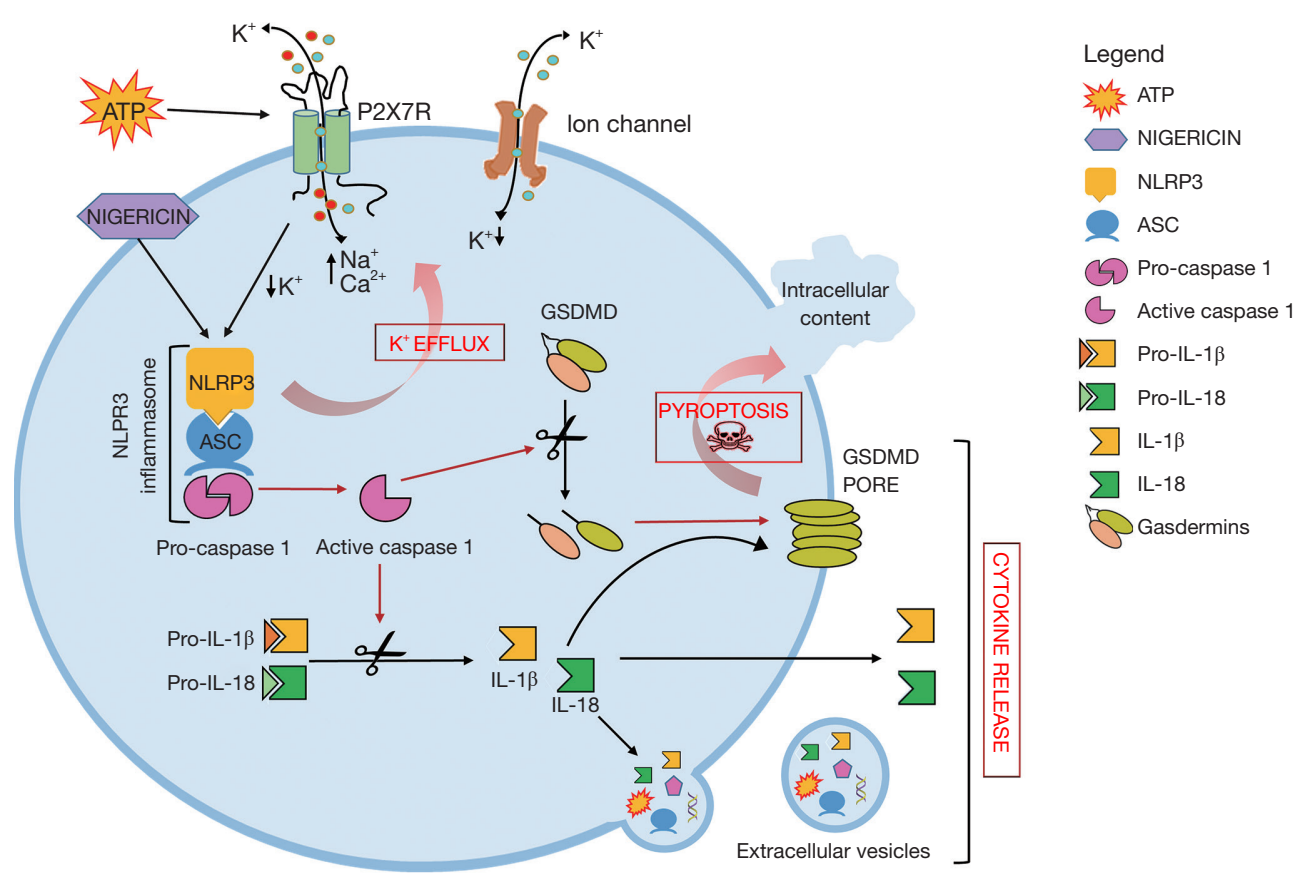

Figure 1 Schematic illustration of NLRP3 inflammasome activation. Various agents including the danger signal ATP and the bacterial toxin nigericin induce potassium efflux and assembly of NLRP3 inflammasome. The formation of NLRP3 protein complex, composed by inactive NLRP3, apoptosis-associated speck-like protein (ASC), and procaspase-1, results in caspase-1 activation. This enzyme cleaves gasdermins at the N-terminus, thus driving the formation of plasma membrane pores and a form of cell death known as pyroptosis. In addition, caspase- 1 cleaves the precursor cytokines pro-IL-1 $\beta$ and pro-IL-18, generating the biologically active cytokines, which are released into the extracellular space as soluble factors through gasdermin-forming pore or extracellular vesicles.

unconventional secretory pathway for the inflammatory cytokines IL-1 $\beta$ and IL-18 (9-12) and to act as vehicles for the transfer of IL-1 $\beta$ transcript between immune cells (13). Recently, EVs released under activation by the transition metal Mn were shown to also transfer the inflammasome adaptor protein ASC between brain resident microglia, thus possibly acting as amplifiers of inflammatory signals in subjects exposed to occupational Mn (welders) (8).

Importantly, EVs diffuse from the brain to the periphery, are present in all body fluids and the analysis of their cargo may provide information on the functional state of donor cells. However, whether circulating EVs may represent biomarkers for inflammasome activation in chronic inflammatory diseases is still largely unexplored.

In a recent issue of Journal of Extracellular Vesicles, Budden and colleagues (14) measured the production of large EVs (or ectosomes) and small EVs (or exosomes) from macrophages exposed to multiple NLRP3 inflammasome activators, and characterized their transcriptional cargo. In line with previous evidence, they found that inflammasome activation enhances production of different classes of EVs, whose size correspond to apoptotic bodies, large EVs and small EVs and identified a specific inflammasome signature in EVs. Specifically, Budden and colleagues profiled the RNA cargo of EVs released upon macrophages stimulation with several NLRP3 activators (LPS + nigericin, LPS + R837 and LPS + IFM-680). Surprisingly enough, they did not use the only well-known physiological activator of the NLRP3 inflammasome (i.e., extracellular ATP!!), and by comparing the RNA content of these EVs to that of EVs released by macrophages primed only with LPS, defined a specific NLRP3 signature. Interestingly, classification of NLRP3 signature transcripts according to their biological function revealed enrichment in ER- and cytoskeletonassociated gene sets in large and small EVs respectively, while mitochondrially encoded mRNAs were preferentially packaged into large EVs under all macrophage stimuli, including LPS only. This suggests that mitochondrially encoded mRNAs are preferentially sorted into large EVs, 


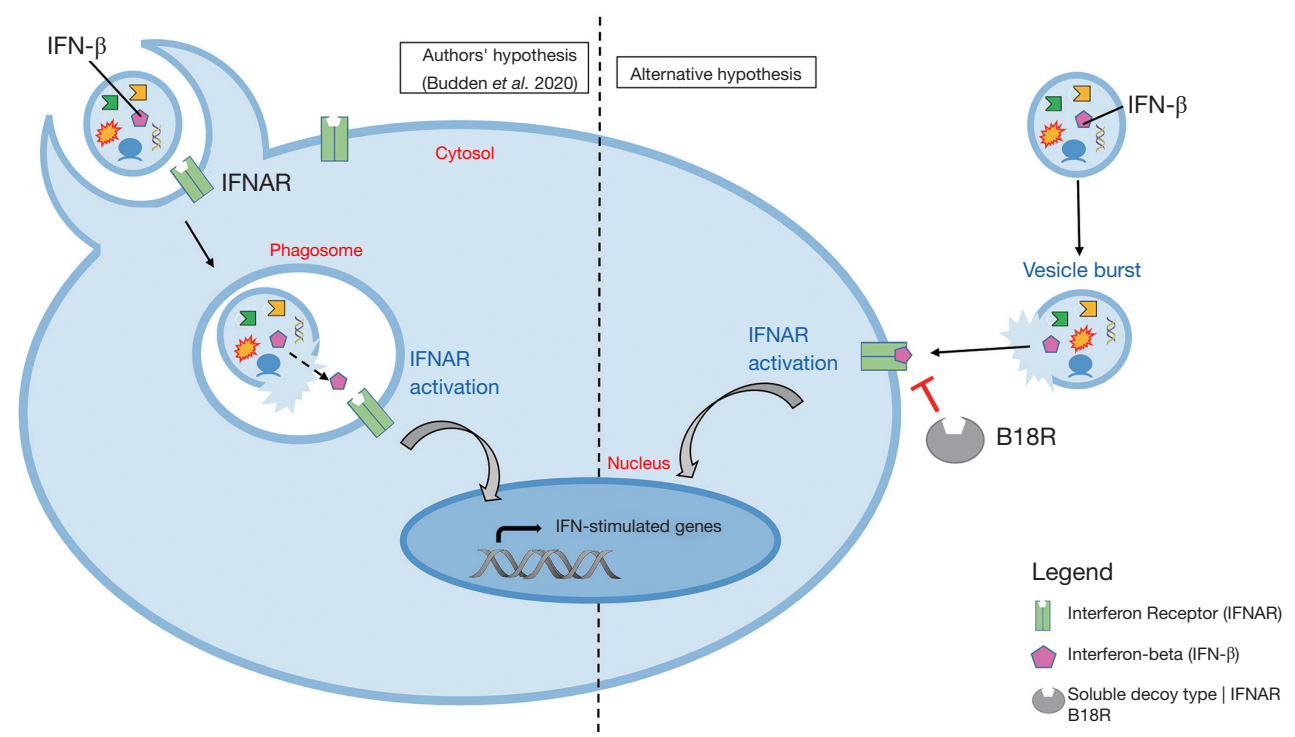

Figure 2 Graphical representation of two hypothesis on IFN-stimulated genes induction following NLRP3 activation. According to Budden and colleagues hypothesis, following extracellular vesicle (EV) phagocytosis by macrophages IFN- $\beta$ packaged within EVs binds and activates its receptor IFNAR into the phagosome, thus inducing IFN-stimulated genes transcription (left side). Alternatively, we suggest that IFN- $\beta$ may become accessible to the extracellular space upon vesicle burst and may induce IFN-stimulated genes by activating IFNAR on cell surface. Indeed, vesicle-mediated induction of IFN-stimulated genes is blocked by the soluble decoy type I IFN receptor B18R (right side).

independently of inflammasome activation, and may therefore be of help in distinguishing large from small EVs on the basis of different molecular composition. Budden and colleagues also profiled the RNA cargo of EVs derived from macrophages stimulated with the inflammasome sensor protein NLRC4 and described the overlap between EV-associated NLRP3 transcripts and transcripts released upon stimulation of NLRC4 as a common inflammasome signature. Thus, these authors suggested that both general and NLRP3-specific inflammasome transcripts may provide insights into the pathogenesis of complex chronic inflammatory diseases, in which the involvement of inflammasome is still largely undefined. Moreover, they suggested that NLRP3 and/or common inflammasome signatures may represent biomarkers for such pathologies. Unfortunately, they did not explore these hypotheses in patients suffering from chronic inflammatory diseases with a likely inflammasome involvement. Therefore, whether the inflammasome signatures identified in macrophagesderived EVs can also be identified in blood-derived EVs and could be used as a biomarker in complex inflammatory diseases still remains to be determined. Future studies are also necessary to verify whether transcripts unique to EVs released upon inflammasome activation may help to understand the contribution of specific inflammasome subtypes to disease pathogenesis.

Analysis of the transcriptome of bystander macrophages exposed to small or large EVs released from NLRP3 inflammasome-triggered macrophages highlighted a strong and selective induction of interferon (IFN)-stimulated genes. However, despite authors' emphasis on the role of EV-mediated mRNA transfer, this response was mainly due to the protein rather than the mRNA cargo. Specifically, the authors convincingly showed that IFN-stimulated genes were induced by IFN- $\beta$ packaged within $\mathrm{EV}$ s via activation of the IFN $\alpha / \beta$ receptor (IFNAR) of recipient macrophages. In fact, large EVs-dependent induction of IFN-stimulated genes was completely inhibited by the soluble decoy type I IFN receptor B18R, and was heat-sensitive, as expected for a protein mediator. IFN- $\beta$ was detected by ELISA in lysed but not intact EVs, suggesting intra-lumen storage rather than association to the EV surface. This raised the question of how the cytokine could interact with IFNAR on recipient macrophages. Given that EVs are taken up by macrophages via phagocytosis, Budden and colleagues hypothesized that the IFN- $\beta$ is discharged into the endosomes where it binds and activate the IFNAR, known to be present in this intracellular compartment (Figure 2). However, whether 
engagement of endosome-located IFNAR per se is sufficient for signaling is not known. Anyway, a similar process was previously suggested to support the anti-inflammatory response of myeloid cells mediated by IL-4 packaged in EVs released by microglia (15). Mechanism of activation of recipient cells by IFN- $\beta$ contained within EVs is clearly puzzling, and left unresolved by the authors. Interestingly, $\mathrm{EV}$-mediated induction of interferon-stimulated genes (ISG) was blocked by the soluble decoy type I IFN receptor B18R, thus suggesting that $\mathrm{EV}$-contained IFN- $\beta$ became somehow accessible to the extracellular phase during the process of EV-recipient macrophage interaction. Since EVs may burst once they reach the target cell, and thus release their content on the their surface (16), it might well be that target cell activation is mediated by interaction of IFN- $\beta$ with the canonical plasma membrane IFNAR, with no role played in this process by the endosome-located IFNAR. Future studies are of course needed to solve this conundrum (Figure 2).

Whichever the mechanism of stimulation of the target cell, these data emphasize the functional relevance of EVs as an unconventional secretory mechanism, as first described already two decades ago for IL- $1 \beta$ by the pioneering work of Surprenant and colleagues (9).

An additional relevant finding of this study is the demonstration that inflammasome-induced EVs have a different impact on bystanders macrophages depending on the recipient cells' priming state. Specifically, inflammasomeinduced EVs negatively regulate inflammasome-dependent responses in unprimed macrophages, in line with the capacity of the IFN- $\beta$ cargo to down-modulate the NLRP3 inflammasome. By contrast, in LPS-primed macrophages, inflammasome-induced EVs serve as an inflammasome activator, and drive IL- $1 \beta$ release. On the basis of these findings and the ability of EVs to circulate between the site of release and the periphery, the authors formulate the intriguing hypothesis that inflammasome-induced EVs have an important function in the overall homeostasis of the inflammatory response by amplifying or inhibiting inflammatory signals at the site of infection or inflammation in the periphery, respectively.

In conclusion, these observations show that the outcome of EV-based intercellular communication depends not only on the EV cargo but also on the functional state of the recipient cells, and provide further demonstration of the unusual plasticity this system of information dispatch in the immune system.

\section{Acknowledgments}

Funding: The work has been supported by Fondazione Italiana Sclerosi Multipla (FISM) (cod. 2018/R/22) and financed or co-financed with the " 5 per mille" public funding.

\section{Footnote}

Provenance and Peer Review: This article was commissioned by the editorial office, $E x R N A$. The article has undergone external peer review.

Conflicts of Interest: The authors have completed the ICMJE uniform disclosure form (available at https://dx.doi. org/10.21037/exrna-21-17). CV was awarded research grants by Horizon 2020 PREMSTEM, Project from Italian Ministry of Health 2018 and Italian Foundation for Multiple Sclerosis, call 2018, and was paid consulting fees to provide her expert opinion to the Scientific Committee of the Italian foundation for Multiple Sclerosis. FV was awarded research grants by Italian Association for Cancer research, Project of National Interest (PRIN), Ministry of Scientific Research and CureAlzheimer USA, and was paid consulting fees to provide his expert opinion on a number of issues related to P2X7R and inflammasome biology. The other author has no conflicts of interest to declare.

Ethical Statement: The authors are accountable for all aspects of the work in ensuring that questions related to the accuracy or integrity of any part of the work are appropriately investigated and resolved.

Open Access Statement: This is an Open Access article distributed in accordance with the Creative Commons Attribution-NonCommercial-NoDerivs 4.0 International License (CC BY-NC-ND 4.0), which permits the noncommercial replication and distribution of the article with the strict proviso that no changes or edits are made and the original work is properly cited (including links to both the formal publication through the relevant DOI and the license). See: https://creativecommons.org/licenses/by-nc-nd/4.0/.

\section{References}

1. de Zoete MR, Palm NW, Zhu S, et al. Inflammasomes. Cold Spring Harb Perspect Biol 2014;6:a016287. 
2. Martinon F, Burns K, Tschopp J. The inflammasome: a molecular platform triggering activation of inflammatory caspases and processing of proIL-beta. Mol Cell 2002;10:417-26.

3. Kovacs SB, Miao EA. Gasdermins: Effectors of Pyroptosis. Trends Cell Biol 2017;27:673-84.

4. Baroja-Mazo A, Martín-Sánchez F, Gomez AI, et al. The NLRP3 inflammasome is released as a particulate danger signal that amplifies the inflammatory response. Nat Immunol 2014;15:738-48.

5. Qu Y, Franchi L, Nunez G, et al. Nonclassical IL-1 beta secretion stimulated by $\mathrm{P} 2 \mathrm{X} 7$ receptors is dependent on inflammasome activation and correlated with exosome release in murine macrophages. J Immunol 2007;179:1913-25.

6. Qu Y, Ramachandra L, Mohr S, et al. P2X7 receptorstimulated secretion of MHC class II-containing exosomes requires the ASC/NLRP3 inflammasome but is independent of caspase-1. J Immunol 2009;182:5052-62.

7. Mouasni S, Gonzalez V, Schmitt A, et al. The classical NLRP3 inflammasome controls FADD unconventional secretion through microvesicle shedding. Cell Death Dis 2019;10:190.

8. Sarkar S, Rokad D, Malovic E, et al. Manganese activates NLRP3 inflammasome signaling and propagates exosomal release of ASC in microglial cells. Sci Signal 2019;12:aat9900.

doi: 10.21037/exrna-21-17

Cite this article as: Verderio C, Lombardi M, di Virgilio F. Differential impact of inflammasome-induced extracellular vesicles on immune cells: key control by the functional state of the recipient cell. ExRNA 2021;3:7.
9. MacKenzie A, Wilson HL, Kiss-Toth E, et al. Rapid secretion of interleukin-1beta by microvesicle shedding. Immunity 2001;15:825-35.

10. Frischer JM, Bramow S, Dal-Bianco A, et al. The relation between inflammation and neurodegeneration in multiple sclerosis brains. Brain 2009;132:1175-89.

11. Pizzirani C, Ferrari D, Chiozzi P, et al. Stimulation of $\mathrm{P} 2$ receptors causes release of IL-1beta-loaded microvesicles from human dendritic cells. Blood 2007;109:3856-64.

12. Gulinelli S, Salaro E, Vuerich M, et al. IL-18 associates to microvesicles shed from human macrophages by a LPS/TLR-4 independent mechanism in response to P2X receptor stimulation. Eur J Immunol 2012;42:3334-45.

13. Verderio C, Muzio L, Turola E, et al. Myeloid microvesicles are a marker and therapeutic target for neuroinflammation. Ann Neurol 2012;72:610-24.

14. Budden CF, Gearing LJ, Kaiser R, et al. Inflammasomeinduced extracellular vesicles harbour distinct RNA signatures and alter bystander macrophage responses. J Extracell Vesicles 2021;10:e12127.

15. Casella G, Colombo F, Finardi A, et al. Extracellular Vesicles Containing IL-4 Modulate Neuroinflammation in a Mouse Model of Multiple Sclerosis. Mol Ther 2018;26:2107-18.

16. Ferrari D, Pizzirani C, Adinolfi E, et al. The P2X7 receptor: a key player in IL-1 processing and release. J Immunol 2006;176:3877-83. 